ARTICLE

DOI: $10.1038 / s 41467-017-01179-y$

\title{
Anomeric memory of the glycosidic bond upon fragmentation and its consequences for carbohydrate sequencing
}

\author{
Baptiste Schindler (10) 1,2,3, Loïc Barnes ${ }^{1,2,3}$, Gina Renois ${ }^{1,2,3}$, Christopher Gray ${ }^{4}$, Stéphane Chambert ${ }^{1,2,5}$, \\ Sébastien Fort ${ }^{6,7}$, Sabine Flitsch ${ }^{4}$, Claire Loison ${ }^{1,2,3}$, Abdul-Rahman Allouche (1) ${ }^{1,2,3}$ \& Isabelle Compagnon ${ }^{1,2,3,8}$
}

Deciphering the carbohydrate alphabet is problematic due to its unique complexity among biomolecules. Strikingly, routine sequencing technologies-which are available for proteins and DNA and have revolutionised biology-do not exist for carbohydrates. This lack of structural tools is identified as a crucial bottleneck, limiting the full development of glycosciences and their considerable potential impact for the society. In this context, establishing generic carbohydrate sequencing methods is both a major scientific challenge and a strategic priority. Here we show that a hybrid analytical approach integrating molecular spectroscopy with mass spectrometry provides an adequate metric to resolve carbohydrate isomerisms, i.e the monosaccharide content, anomeric configuration, regiochemistry and stereochemistry of the glycosidic linkage. On the basis of the spectroscopic discrimination of MS fragments, we report the unexpected demonstration of the anomeric memory of the glycosidic bond upon fragmentation. This remarkable property is applied to de novo sequencing of underivatized oligosaccharides.

\footnotetext{
${ }^{1}$ Université de Lyon, F-69622 Lyon, France. ${ }^{2}$ Université Lyon 1, Villeurbanne, France. ${ }^{3}$ Institut Lumière Matière, UMR5306 Université Lyon 1-CNRS, Université de Lyon, 69622 Villeurbanne Cedex, France. ${ }^{4}$ School of Chemistry \& Manchester Institute of Biotechnology, The University of Manchester, 131 Princess Street, Manchester M1 7DN, UK. ${ }^{5}$ Laboratoire de Chimie Organique et Bioorganique, INSA Lyon, CNRS, UMR5246, ICBMS, Bât. J. Verne, 20 Avenue A. Einstein, 69621 Villeurbanne Cedex, France. ${ }^{6}$ Université de Grenoble Alpes, CERMAV, F-38000 Grenoble, France. ${ }^{7}$ CNRS, CERMAV, F-38000 Grenoble, France. ${ }^{8}$ Institut Universitaire de France IUF, 103 Blvd St Michel, 75005 Paris, France. Correspondence and requests for materials should be addressed to I.C. (email: isabelle.compagnon@univ-lyon1.fr)
} 
T he first principles of protein and DNA sequencing, which have been established between 1952 and 1977, have revolutionised modern biology and have even become amazingly popular among the non-scientific public. Yet, it is striking that routine, high-throughput sequencing technology is not available for oligosaccharides. This lack of carbohydrateoriented structural tools, which is identified as a critical impairment to the full development of glycosciences ${ }^{1,2}$, is essentially due to their unique molecular complexity among natural biopolymers.

The basic concept of tandem-mass spectrometry (MS) analysis of a (bio-)polymer sequence rests on two foundational hypothesis: the molecular structure of fragmentation products must be translatable to the parent polymer through retention of structural features of the precursor; and one must have available an effective metric offering a sufficient level of structural detail of fragments to retrieve the structure of the precursor. These pre-requisites are verified for proteins and nucleic acids, which are linear biopolymers formed from a relatively small pool of often non-isomeric monomers, allowing the parent sequence to be deduced from $\mathrm{m} / \mathrm{z}$ alone $e^{3}$. The case of carbohydrates is more complex as both the monomer composition (most of which are epimers of one another), and the regiochemistry and stereochemistry of the inter-glycosidic linkages must be identified. Moreover, these isomeric carbohydrate structural features are not readily resolved using traditional MS techniques ${ }^{4}$. To date, the preservation of these carbohydrate structural features upon fragmentation is poorly understood. Domon and Costello ${ }^{5}$ proposed a nomenclature for MS carbohydrate fragments, but their exact molecular structure was not verified, largely due to the lack of a universal metric with sufficient structural resolution to disambiguate all carbohydrate isomerisms. a Epimers/diastereoisomers

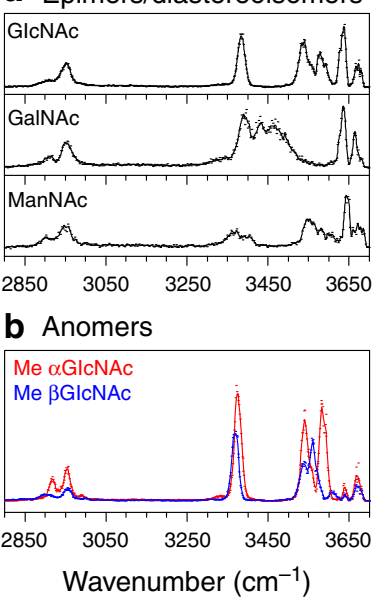

C Disaccharides
Gal

GIcNAc
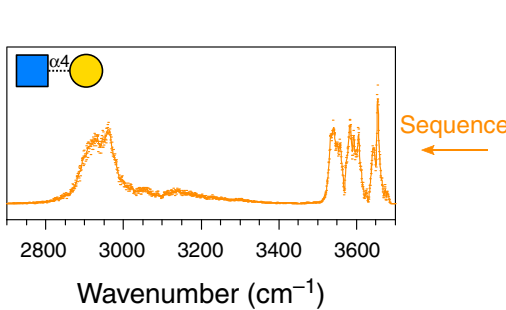
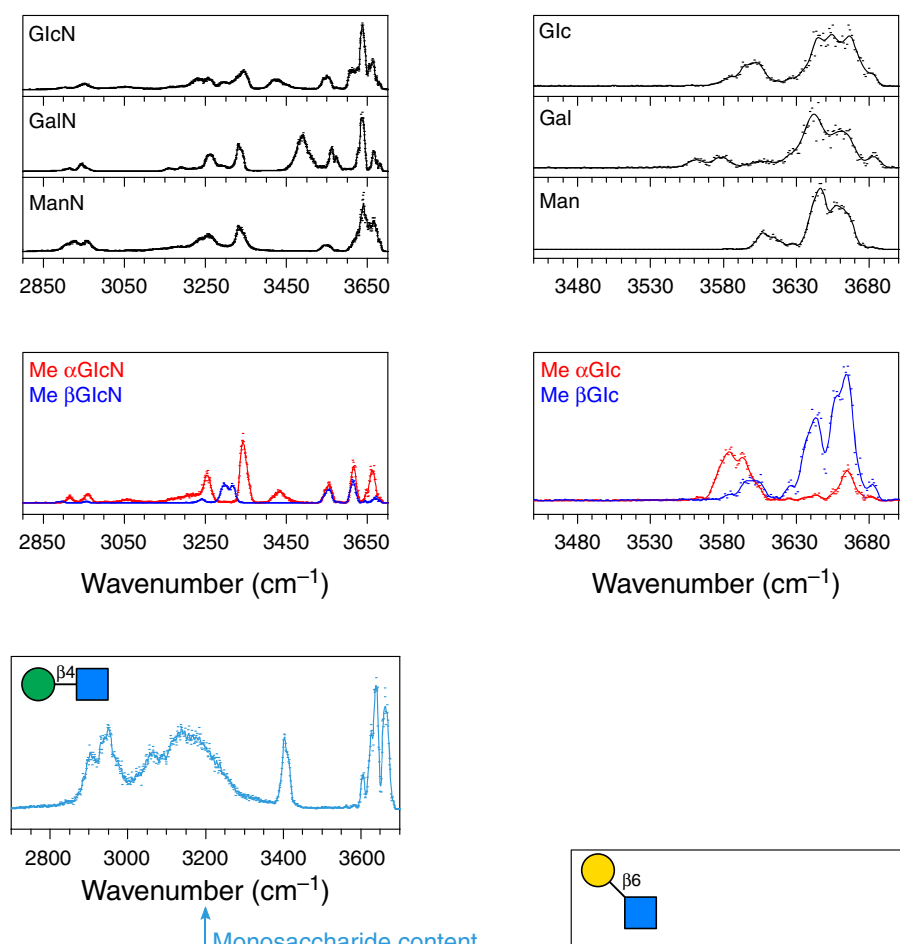

$\begin{array}{lllll}3480 & 3530 & 3580 & 3630 & 3680\end{array}$

Monosaccharide content
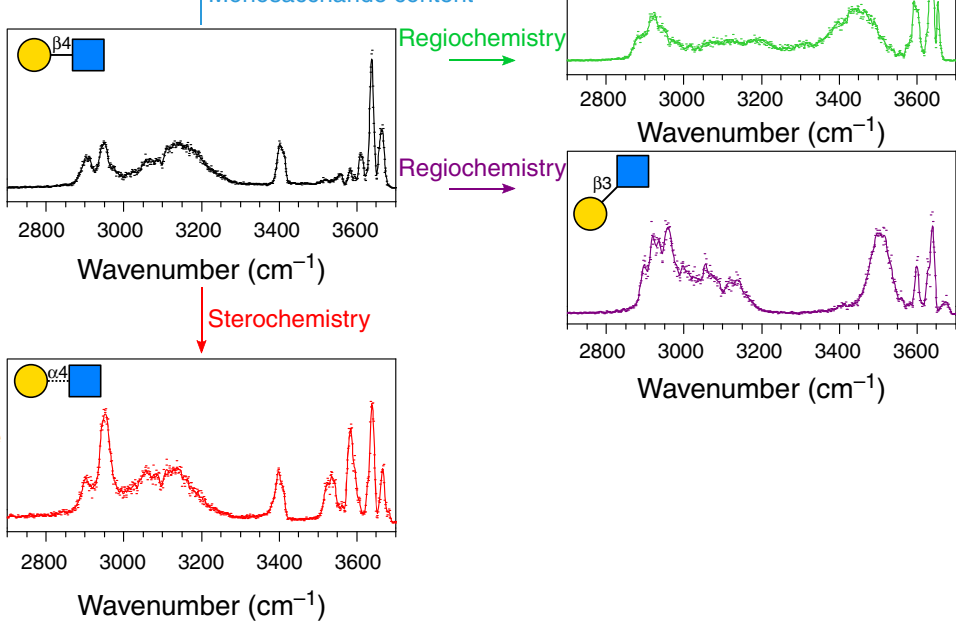

Fig. 1 IRMPD signatures of monosaccharide and disaccharide standards. a $\mathrm{N}$-acetylhexosamines, hexosamines and hexoses. $\mathbf{b}$ Individual methyl-blocked anomers of $\mathrm{N}$-acetylglucosamine, glucosamine and glucose. c Isomers of GalGlcNAc: Gal $\beta 1,4 \mathrm{GlcNAc}$ (black); Man $\beta 1,4 \mathrm{GlcNAc}$ (blue); Gal $\beta 1,3 \mathrm{GlcNAc}$

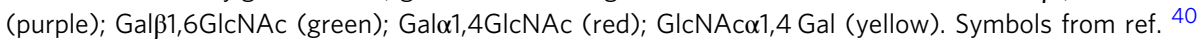


a Domon \& Costello nomenclature

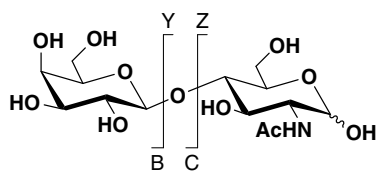

b Monosaccharide content

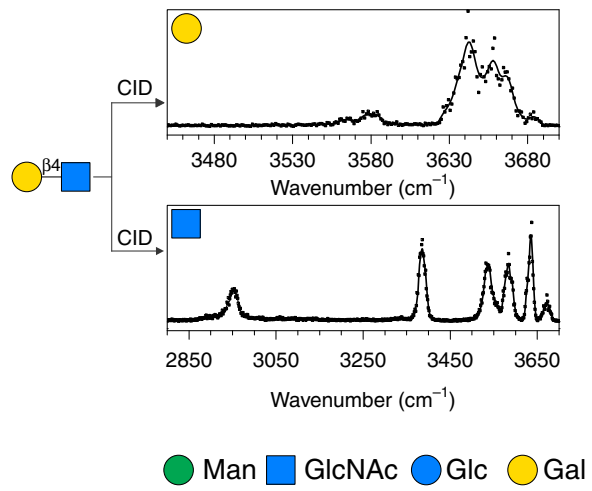

C Anomeric memory

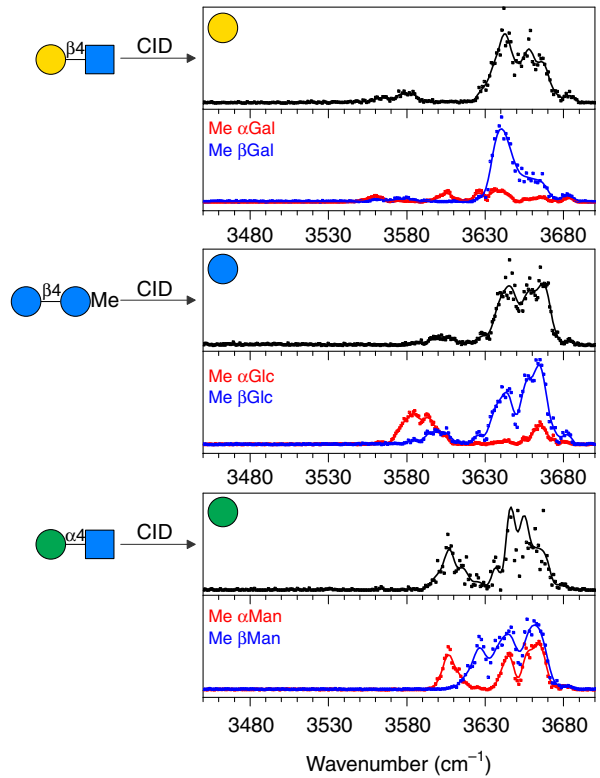

Fig. 2 Spectroscopic analysis of disaccharides fragments. a Nomenclature of carbohydrates MS fragments, after Domon and Costello ${ }^{5}$. b Monosaccharide fragments of Gal $\beta 1,4 \mathrm{GlcNAc}$. c Non-reducing fragments of Gal $\beta 1,4 \mathrm{GlcNAc}$; Glc $\beta 1,4 \mathrm{GlcMe}$ and Man $\alpha 1,4 \mathrm{GlcNAc}$. Methyl-anomers of Gal, Glc and Man are shown for comparison. Symbols from ref. ${ }^{40}$

Owing to these limitations, structural elucidation of unknown oligosaccharides from biological sources largely relies on NMR, which may imply months of extraction and purification of a sufficient amount of sample, with possible alteration of labile functional modifications. Alternatively, mass spectrometric analysis applies to natural samples but does not readily resolve all carbohydrate isomerisms. Whereas MS/MS analysis of cross-ring fragments is useful to resolve regiochemistry, the stereochemistry and the monosaccharide content remain ambiguous by MS analysis alone. This has stimulated considerable efforts to explore integrated hyphenated approaches. Such approaches aim at providing direct structural information on mass-selected ions without the recourse to chemical derivatisation of the analyte, or to a high level of chemical purification. In particular, ion mobility spectrometry (IMS) and field asymmetric ion mobility spectrometry (FAIMS) separation of isomeric carbohydrates were explored since 1997 using home-built FAIMS-MS or IMS-MS instruments $^{6-11}$. More recently, the formidable potential of commercial IMS-MS instruments has been demonstrated ${ }^{12-16}$. Alternatively, Bendiak and colleagues ${ }^{17}$ proposed an approach based on IRMPD spectroscopy (infrared multiple photon dissociation) to explore saccharides isomers in the free-electron laser spectral range $(5-10 \mu \mathrm{m})$; whereas Pohl and colleagues ${ }^{18,19}$ have reported monosaccharide identification using kinetic measurements. So far however, none of these methods could resolve simultaneously all carbohydrates isomerisms.

Although the question of the universal metric for carbohydrates remains open, MS sequencing also requires that the structural properties of an oligomer are preserved upon fragmentation and can be retrieved via the structural analysis of its fragments. The Domon and Costello nomenclature for carbohydrate MS fragments suggests that monosaccharides fragments of type $\mathrm{C}$ and $\mathrm{Y}$ are intact monosaccharide units; and $\mathrm{C}$ fragments bear the anomeric configuration of the glycosidic bond of the precursor. This was however never formally demonstrated and these hypotheses need to be verified for the purpose of carbohydrate sequencing.
In this context, few groups have reported ion mobility exploration of carbohydrates fragments. Pagel in particular reported the identification of structurally informative patterns in glycans via the analysis of trisaccharide fragments ${ }^{20,21}$. Others reported collision cross sections of monosaccharide fragments, but the results were difficult to relate to the collision cross sections of the corresponding monosaccharide standards ${ }^{13}, 22,23$. Recently, we have reported that B and C fragments of glucose containing disaccharides exhibit precursor-dependant profiles ${ }^{24}$. So far, reliable discrimination of monosaccharide fragments and the identification of their anomeric configuration are limited by structural resolution of ion mobility spectrometry. As a direct consequence, the fundamental hypothesis underlying top-down carbohydrate analysis, that is the anomeric memory of $\mathrm{C}$ fragments suggested by Domon and Costello, remains to be investigated.

Here, we present the spectroscopic fingerprint in the $3 \mu \mathrm{m}$ spectral range as a versatile carbohydrate metric. Its refined structural resolution allows simultaneous resolution of monosaccharide isomers, regio-isomers and stereo-isomers in an integrated instrument combining MS and IR spectroscopy. Using the resolving power of this metric, we explore the structure of carbohydrate fragments and we report the validated observation of anomeric memory of the glycosidic bond within C-fragments. Finally, we illustrate the potential of this remarkable property for de novo carbohydrate sequencing, with the full characterisation (i.e. degree of polymerisation, degree of acetylation and pattern of acetylation) of a crude sample of chito-oligosaccharides.

\section{Results}

Carbohydrate isomerisms resolution using IRMPD fingerprint. In traditional IR spectroscopy, the $3 \mu \mathrm{m}$ spectral range is of little molecular specificity due to the interferences with aqueous solvents. For carbohydrates in the gas phase however, this spectral range is highly specific, as established by the pioneering work of Simons ${ }^{25}$ on jet-cooled, synthetically phenyl-grafted 
monosaccharides and oligosaccharides ${ }^{26,27}$. With the integration of IR spectroscopy to mass spectrometry (IRMPD spectroscopy), the applicability of this highly relevant spectral range has been generalised to non-derivatised carbohydrates. Several groups have reported diagnostic fingerprints of monosaccharides isomers in this spectral range, including hexoses ${ }^{28,29}$, hexuronic acids ${ }^{30,31}$, $\mathrm{N}$-acetyhexosamines 32,33 , and sulfated and phosphorylated monosaccharides ${ }^{34-36}$. Recently, we have also reported the spectroscopic identification of oligosaccharide isomers ${ }^{36,37}$.

Here we further generalise the $3 \mu \mathrm{m}$ fingerprint as an adequate metric to resolve carbohydrate isomerisms by briefly reviewing the gas phase IR signatures of a range of monosaccharide and disaccharide standards.

As shown in Fig. 1a, epimers and/or diastereoisomers of the most abundant natural hexoses, $\mathrm{N}$-acetylhexosamines and hexosamines can unambiguously be discriminated by their spectroscopic signatures. It is possible to further assign the spectroscopic features to individual hydroxyl and $\mathrm{NH}$ groups and to carry out complete conformational analysis by comparison with quantum chemistry simulations (Supplementary Fig. 1). Figure $1 \mathrm{~b}$ shows the reference spectra of pairs of anomers (blocked by 1-O-methylation), which display distinctive signatures. Interestingly, these data can be used to identify the contributions of both anomers to the spectrum of a natural monosaccharide (Supplementary Fig. 2). The IR fingerprint is also diagnostic for the stereochemistry of the glycosidic bond, as shown in Fig. 1c for Gal $\beta 1,4$ GlcNAc (black trace) and Gal $\alpha 1,4 \mathrm{GlcNAc}$ (red trace) and for an inversion of sequence
(GlcNAc $\alpha 1,4 \mathrm{Gal}$, yellow trace). A change of monosaccharide content-known to be undetectable by ion mobility spectrometry ${ }^{12}$ - results in a small, yet observable change of the IR fingerprint around $3600 \mathrm{~cm}^{-1}$ (blue trace). Finally, the regioisomers of $\beta$-linked GalGlcNAc have well-resolved IR signatures (purple, black and green traces), in contrast with recent ion mobility data reported by Pagel and colleagues ${ }^{21}$ Thus, IRMPD spectroscopy offers an efficient alternative to more elaborate spectroscopic schemes-such as this initially proposed by John Simons, or the cryogenic schemes recently proposed by Rizzo and colleagues $^{38}$ and Pagel and colleagues ${ }^{39}$ for the distinction of carbohydrate isomers.

These results highlight the complementarity of IR spectroscopy with ion mobility spectrometry and qualify the IR fingerprint in the $3 \mu \mathrm{m}$ spectral range as a powerful metric for structural characterisation of carbohydrates. Nevertheless, two limitations of this approach for glycoanalysis can be anticipated. First, the identification of an oligosaccharide will rely on the availability of the corresponding standard. Second, the structural resolution of the MS/IR fingerprint tends to decrease as the size of the oligosaccharide increases ${ }^{37}$. To overcome these two limitations, it is essential to adopt a top-down sequencing approach, as described in the next section.

Spectroscopic analysis of MS fragmentation products. IR spectroscopy integrated to MS offers the possibility to perform spectroscopic analysis building block by building block after

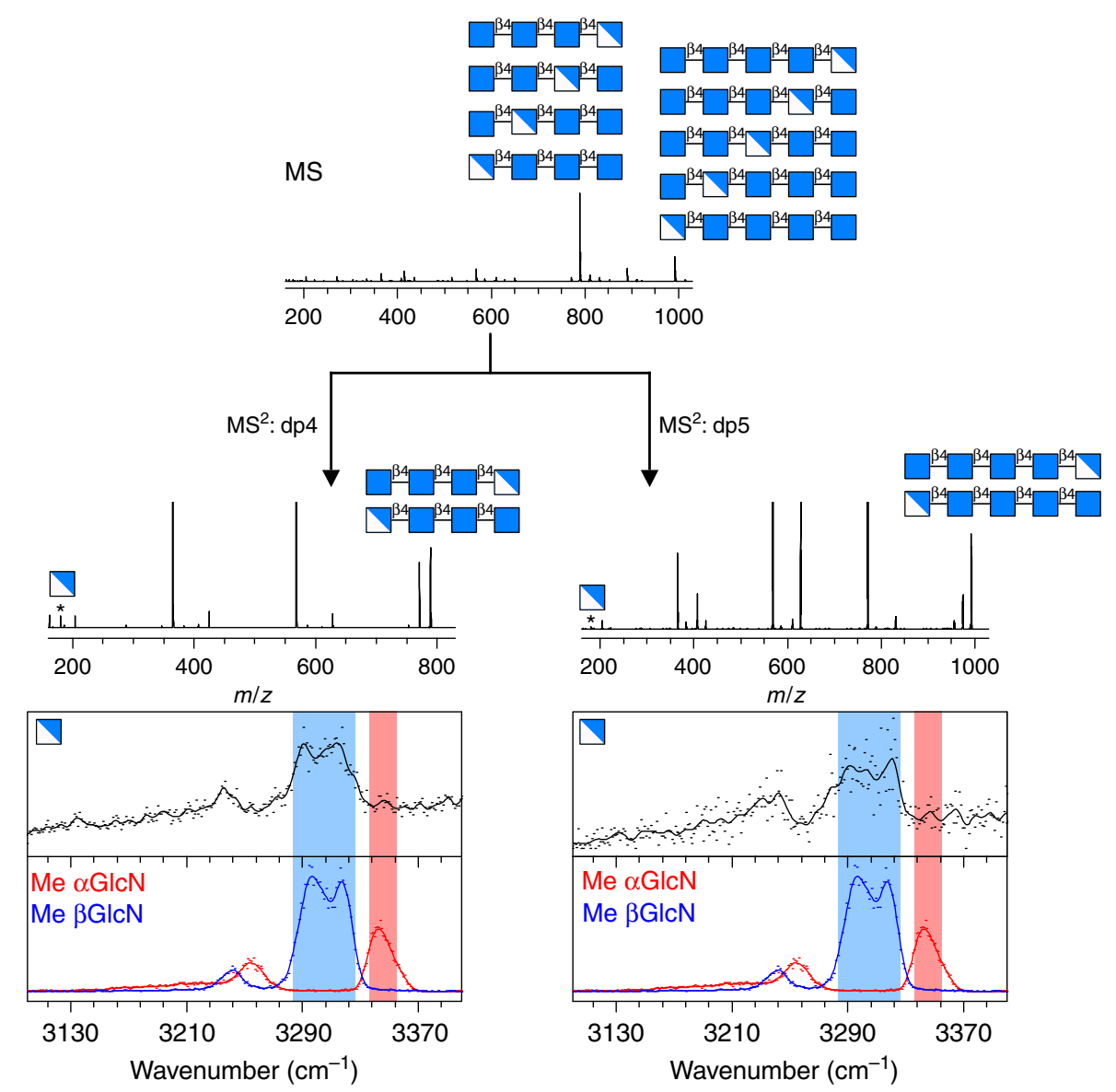

Fig. 3 Illustration of the carbohydrate-sequencing procedure in the case of a crude mixture of chito-oligosaccharides: the degree of polymerisation and the fraction of acetylation are resolved by MS; the set of candidate sequences is reduced by MS/MS analysis; the sequence is fully resolved by spectroscopic analysis of the GIcN fragment. Symbols from ref. 40 
fragmentation by MS/MS. To verify that sequence information is conserved within the MS fragments proposed by Domon and Costello (Fig. 2a), we assessed their structure by comparison of their spectroscopic signatures with the reference spectra of monosaccharides standards.

Elucidation of the monosaccharide content. MS/MS analysis of Gal 1 1,4GlcNAc shows two monosaccharide moieties: a hexose (C fragment) and an $\mathrm{N}$-acetylhexosamine (Y fragment), but does not readily indicate their nature. To resolve the monosaccharide content, the IR fingerprints of these two MS fragments are measured, as shown in Fig. 2b, and compared with the reference IR signatures of hexose and $\mathrm{N}$-acetylhexosamine standards shown in Fig. 1a. The fingerprints of the $\mathrm{C}$ and $\mathrm{Y}$ fragments unambiguously match those of galactose and $\mathrm{N}$-acetylglucosamine, respectively. This validates that the monosaccharide content of an oligosaccharide can be retrieved by IR analysis of its fragments. Remarkably, identification only relies on the comparison with a reduced database of monosaccharide standards and does not require a-potentially much larger-set of disaccharide references.

Anomeric memory of the glycosidic bond upon fragmentation. A closer examination of the galactose $C$ fragment reveals that its fingerprint is not exactly identical to that of the galactose standard shown in Fig. 1a. Indeed, the small band at $3605 \mathrm{~cm}^{-1}$ present in the spectrum of the standard is absent in the spectrum of the fragment. Further comparison with the reference fingerprints of the individual methyl-blocked anomers $\alpha \mathrm{Gal}$ and $\beta \mathrm{Gal}$ is shown in Fig. 2c. It appears that this feature at $3605 \mathrm{~cm}^{-1}$ is diagnostic of the $\alpha$ anomer, and its absence in the spectrum of the $\mathrm{C}$ fragment indicates that the galactose moiety retains a pure $\beta$ character after fragmentation of the glycosidic bond. Similarly, Fig. $2 c$ shows that the glucose moiety of Glc $\beta 1,4 \mathrm{GlcMe}$ retains a pure $\beta$ character (by comparison with methyl-blocked $\alpha$ Glc and $\beta$ Glc standards); and the mannose moiety of Man $\alpha 1,4$ GlcNAc retains a pure $\alpha$ character (by comparison with methyl-blocked $\alpha$ Man and $\beta$ Man standards).

This observation constitutes evidence that the anomeric configuration of monosaccharide fragments holds the memory of the stereochemistry of the glycosidic bond. The reducing monosaccharide is the exception: it displays both $\alpha$ and $\beta$ characters, as seen in the previous section. As a consequence of this important property, both the monosaccharide content and the stereochemistry of the glycosidic bond can be resolved by spectroscopic analysis of the monosaccharide fragments, which is an essential finding in the prospective of carbohydrate sequencing.

Application to carbohydrate sequencing. By extension, the monosaccharide content and the stereochemistry of an oligosaccharide can be elucidated by spectroscopic analysis of its monosaccharide fragments. To that end, IR spectroscopy is performed in a mass spectrometer equipped with an ion trap with $\mathrm{MS}^{\mathrm{n}}$ capability. In this configuration monosaccharide fragments are produced sequentially from the end and the order of the sequence is preserved in the process ${ }^{41}$. Thus, step-by-step spectroscopic analysis of the monosaccharide content and stereochemistry can be used to resolve the carbohydrate sequence.

As an illustration, we apply this sequencing approach to the resolution of chito-oligosaccharide sequences in a crude sample. This class of linear oligosaccharides consists of $\beta 1,4$-linked GlcNAc and GlcN units. A comprehensive description of their structure includes their degree of polymerisation (DP), their fraction of acetylation (FA) and their pattern of acetylation (PA).
Although the DP and FA can be resolved by MS analysis, the elucidation of the PA remains an obstacle and requires chemical labelling of the reducing end ${ }^{42}$. It was also shown that the PA can not be readily resolved by $\mathrm{NMR}^{43}$. Although the stereochemistry of this class of saccharides is well-know, we show here that the anomeric memory can be advantageously used to resolve the pattern of acetylation, without the need for purification or chemical labelling of the sample.

The MS spectrum of a crude sample of chito-oligosaccharides is shown in Fig. 3. The spectrum displays two main peaks at 789 and $992 \mathrm{~m} / z$, which correspond to singly deacetylated tetrasaccharides and pentasaccharides, respectively. The degree of polymerisation and the fraction of acetylation are thus readily resolved. The patterns of acetylation remain unknown however: four and five candidate sequences are possible for the tetrasaccharide (dp4) and the pentasaccharide (dp5), respectively. Further MS/MS analysis of these two species shows a GlcN fragment at $180 \mathrm{~m} / z$ (marked with stars in Fig. 3), which indicates that both oligosaccharides are deacetylated at one end. On the basis of this additional information, only two candidate sequences remain. To fully resolve the sequence, the origin of the GlcN fragment (reducing or non-reducing end) must be elucidated. To that end, it is necessary to identify its anomeric configuration. Indeed, the reducing end monosaccharide would show both $\alpha$ and $\beta$ characters (such as the GlcN standard shown in Fig. 1), whereas the non-reducing end monosaccharide would retain a pure $\beta$ configuration after fragmentation of the $\beta$ bond. The IR fingerprints of the GlcN fragments are displayed in Fig. 3 and compared with the relevant standards (methyl-blocked $\alpha \mathrm{GlcN}$ and $\beta \mathrm{GlcN}$ ) in the highly anomeric-specific spectral region $3100-3400 \mathrm{~cm}^{-1}$. It shows clearly that the fingerprints of the GlcN fragments match this of the $\beta G l c N$ standard: the $\beta$ diagnostic features (highlighted in blue) are present, whereas the $\alpha$ diagnostic feature (highlighted in red) is absent. This indicates that both species present in the sample are deacetylated at the non-reducing end. By combining MS and spectroscopic structural information, the sequences of the oligosaccharides present in the crude sample are thus fully resolved.

\section{Discussion}

We have demonstrated that the IRMPD signature in the $3 \mu \mathrm{m}$ spectral range is a powerful metric to resolve carbohydrate isomerisms, and we highlight its complementarity with the structural resolution offered by ion mobility spectrometry. IRMPD allows for a further spectroscopic exploration of the structure of MS fragments. Comparison of the IR fingerprints of monosaccharide fragments with monosaccharide standards reveals two remarkable properties: the monosaccharide content can be elucidated and the stereochemistry of the glycosidic bond is retained after fragmentation.

On the basis of these findings, we propose the basic principles of a generic method for oligosaccharide sequencing. As an example of application of the method, the sequences of chitooligosaccharides were resolved directly from a crude sample, without chemical labelling, and without the recourse to chitooligosaccharide standards.

Compared to other MS-based sequencing strategies, the proposed method has three advantages. First, it does not require any chemical derivatisation of the analyte, thus saving considerable sample preparation. Second, a minimal database of monoaccharide standards is sufficient to perform a spectroscopic identification of the monosaccharide content and stereochemistry of any oligosaccharide. As such, this is a de novo method, in contrast with MS/MS analysis, which requires reference MS/MS data for the corresponding standard and generally involves the 
availability of a large set of candidates. Finally, adding a spectroscopic dimension to mass spectrometry combines the advantages of MS-sequencing strategies with an unparalleled resolution of carbohydrate isomerisms in an integrated instrument. The method can thus be used either standalone or complement to other sequencing strategies when structural ambiguities remain. With a typical analysis time of $10 \mathrm{~min}$ per monosaccharide, we expect that this hybrid analytical solution will have an immediate and broad impact in glycoanalysis.

\section{Methods}

MS is performed using a quadrupole ion trap mass spectrometer (LCQ Thermo Finnigan) coupled with electrospray ionisation source. Protonated hexosamines and $N$-acetylhexosamines are formed from $250 \mu \mathrm{M} \mathrm{H}_{2} \mathrm{O}: \mathrm{MeOH}$ (50:50) solutions. Lithium-hexoses complexes are formed from $250 \mu \mathrm{M} \mathrm{H}_{2} \mathrm{O}: \mathrm{MeOH}$ (50:50) with 50 $\mu \mathrm{M} \mathrm{LiCl}$ solutions.

The mass spectrometer was modified for IRMPD spectroscopy to allow injection of a tuneable IR laser beam (IR OPO/OPA Laservision pumped with a $10 \mathrm{~Hz}$ YAG Continuum Surelite) inside of the ion trap to perform the IRMPD spectroscopy. When the IR wavelength is resonant with a vibrational mode of the mass-selected ions, several photons are absorbed, thus increasing the internal energy of the ion, and eventually resulting in photofragmentation. Three photofragmentation mass spectra are averaged for each wavelength and the photofragmentation yield (area fragment peak/area parent peak ratio in log scale, no unit) is plotted as a function of the wavelength without further data processing. All data points shown thorough the manuscript were obtained using the same procedure. Finally, a trend line (5points Fourier Transform rolling averaging) is added to guide the eye. The assessment of the spectral match between the fingerprint of a fragment and the library of reference IRMPD spectra of standards is done by visual inspection.

Sample preparation and computational chemistry are detailed in Supplementary Methods.

Data availability. All other data is available from the authors upon reasonable request. Besides, the instrument is available via the IROGLYPH platform.

Received: 2 June 2017 Accepted: 24 August 2017

Published online: 17 October 2017

\section{References}

1. National Research Council (US) Committee on Assessing the Importance and Impact of Glycomics and Glycosciences. Transforming Glycoscience A Roadmap for the Future. (National Academic Press, Washington, DC, 2012).

2. EGSF, IBCarb Network \& European Science Foundation. A Roadmap for Glycosciences in Europe. (2014).

3. Roepstorff, P. \& Fohlman, J. Proposal for a common nomenclature for sequence ions in mass spectra of peptides. Biomed. Mass Spectrom. 11, 601-601 (1984).

4. Zaïa, J. Mass spectrometry of oligosaccharides. Mass Spectrom. Rev. 23, 161-227 (2004).

5. Domon, B. \& Costello, C. E. A systematic nomenclature for carbohydrate fragmentations in FAB-MS/MS spectra of glycoconjugates. Gycoconjugate J. 5, 397-409 (1988).

6. Liu, Y. \& Clemmer, D. E. Characterizing oligosaccharides using injected-ion mobility/mass spectrometry. Anal. Chem. 69, 2504-2059 (1997).

7. Gabryelski, W. \& Frose, K. L. Rapid and sensitive differentiation of anomers, linkage, and position isomers of disaccharides using high-field asymmetric waveform ion mobility spectrometry (FAIMS). J. Am. Chem. Soc. Mass Spectrom. 14, 265-277 (2003).

8. Clowers, B. H., Dwivedi, P., Steiner, W. E. \& Bendiak, B. J. Separation of sodiated isobaric disaccharides and trisaccharides using electrospray ionizationatmospheric pressure ion mobility-time of flight mass spectrometry. Am. Chem. Soc. Mass Spectrom. 16, 660-669 (2005).

9. Williams, J. P. Characterization of simple isomeric oligosaccharides and the rapid separation of glycan mixtures by ion mobility mass spectrometry. Int. J. Mass Spectrom. 298, 119-127 (2010).

10. Dwivedi, P., Bendiak, B., Clowers, B. H. \& Hill, H. H. Jr. Rapid resolution of carbohydrate isomers by electrospray ionization ambient pressure ion mobility spectrometry-time-of-flight mass spectrometry (ESI-APIMS-TOFMS). J. Am. Soc. Mass Spectrom. 18, 1163-1175 (2007).

11. Gaye, M. M., Nagy, G., Clemmer, D. E. \& Pohl, N. L. B. Multidimensional analysis of 16 glucose isomers by ion mobility spectrometry. Anal. Chem. 88, 2335-2344 (2016).
12. Hofmann, J., Hahm, H. S., Seeberger, P. H. \& Pagel, K. Identification of carbohydrate anomers using ion mobility-mass spectrometry. Nature 526, 241-244 (2015)

13. Both, P. et al. Discrimination of epimeric glycans and glycopeptides using IM-MS and its potential for carbohydrate sequencing. Nat. Chem. 6, 65-74 (2014).

14. Li, H. et al. Resolving structural isomers of monosaccharide methyl glycosides using drift tube and traveling wave ion mobility mass spectrometry. Anal. Chem. 84, 3231-3239 (2012).

15. Hofmann, J. \& Pagel, K. Glycan analysis by ion mobility-mass spectrometry. Angrew Chem. lnt. Ed. 56, 8342-8349 (2017).

16. Pagel, K. \& Harvey, D. J. Ion mobility-mass spectrometry of complex carbohydrates: Collision cross sections of sodiated N-linked glycans. Anal. Chem. 85, 5138-5145 (2013).

17. Polfer, N. C. et al. Differentiation of isomers by wavelength-tunable infrared multiple-photon dissociation-mass spectrometry: application to glucosecontaining disaccharides. Anal. Chem. 78, 670-679 (2006).

18. Nagy, G. \& Pohl, N. L. B. Complete hexose isomer identification with mass spectrometry. J. Am. Soc. Mass Spectrom. 26, 677-685 (2015).

19. Nagy, G. \& Pohl, N. L. B. Monosaccharide identification as a first step toward de novo carbohydrate sequencing: mass spectrometry strategy for the identification and differentiation of diastereomeric and enantiomeric pentose isomers. Anal. Chem. 87, 4566-4571 (2015).

20. Hinneburg, $\mathrm{H}$. et al. Distinguishing $\mathrm{N}$-acetylneuraminic acid linkage isomers on glycopeptides by ion mobility-mass spectrometry. Chem. Commun. 52, 4381-4384 (2016).

21. Hofmann, J. et al. Identification of Lewis and blood group carbohydrate epitopes by ion mobility-tandem-mass spectrometry fingerprinting. Anal. Chem. 89, 2318-2325 (2017).

22. Gaye, M. M., Kurulugama, R. \& Clemmer, D. E. Investigating carbohydrate isomers by IMS-CID-IMS-MS: precursor and fragment ion cross-sections. Analyst 140, 6922-6932 (2015).

23. Li, H., Bendiak, B., Siems, W. F., Gang, D. R. \& Hill, H. H. Jr. Ion mobility mass spectrometry analysis of isomeric disaccharide precursor, product and cluster ions. Rapid Commun. Mass Spectrom. 27, 2699-2709 (2013).

24. Gray, C. J. et al. Bottom-up elucidation of glycosidic bond stereochemistry. Anal. Chem. 89, 4540-4549 (2017).

25. Cocinero, E. J., Carçabal, P., Vaden, T. D., Simons, J. P. \& Davis, B. G. Sensing the anomeric effect in a solvent-free environment. Nature 469, 76-79 (2011).

26. Jockush, R. A. et al. Probing the glycosidic linkage: UV and IR ion-dip spectroscopy of a lactoside. J. Am. Chem. Soc. 126, 5709-5714 (2004).

27. Çarçabal, P. et al. Hydrogen bonding and cooperativity in isolated and hydrated sugars: mannose, galactose, glucose, and lactose. J. Am. Chem. Soc. 127, 11414-11425 (2005).

28. Pearson, W. L. III et al. Differentiation of rubidiated methyl-D-glycoside stereoisomers by infrared multiple-photon dissociation spectroscopy in the O-H and C-H stretching regions. J. Phys. Chem. B 119, 12970-12981 (2015).

29. Hernandez, O., Isenberg, S., Steinmetz, V., Glish, G. L. \& Maitre, P. Probing mobility-selected saccharide isomers: Selective ion-molecule reactions and wavelength-specific IR activation. J. Phys. Chem. A 119, 6057-6064 (2015).

30. Schindler, B. et al. MS/IR, a new MS-based hyphenated method for analysis of hexuronic acid epimers in glycosaminoglycans. Glycoconjugate J. 34, 421-425 (2016).

31. Cagmat, E. B. et al. Vibrational signatures of metal-chelated monosaccharide epimers: Gas-phase infrared spectroscopy of $\mathrm{Rb}^{+}$-tagged glucuronic and iduronic acid. Phys. Chem. Chem. Phys. 12, 3474-3479 (2010).

32. Barnes, L., Schindler, B., Chambert, S., Allouche, A. R. \& Compagnon, I. Conformational preferences of protonated $\mathrm{N}$-acetylated hexosamines probed by infrared multiple photon dissociation (IRMPD) spectroscopy and ab initio calculations. Intl. J. Mass Spectrom. https://doi.org/10.1016/j.ijms.2017.05.005 (2017).

33. Tan, Y. et al. Vibrational signatures of isomeric lithiated N-acetyl-Dhexosamines by Gas-phase infrared multiple-photon dissociation (IRMPD) spectroscopy. J. Am. Soc. Mass Spectrom. 28, 539-550 (2016).

34. Schindler, B. et al. Distinguishing isobaric phosphated and sulfated carbohydrates by coupling of mass spectrometry with gas phase vibrational spectroscopy. Phys. Chem. Chem. Phys. 16, 22131-22138 (2014).

35. Barnes, L. et al. Anharmonic simulations of the vibrational spectrum of sulfated compounds: application to the glycosaminoglycan fragment glucosamine 6sulfate. Phys. chem. Chem. Phys. 17, 25705-25731 (2015).

36. Schindler, B. et al. IRMPD spectroscopy sheds new (infrared) light on the sulfate pattern of carbohydrates. J. Phys. Chem. A. 121, 2114-2120 (2017).

37. Wattjes, J., Schindler, B., Trombotto, S., Moerschbacher, D. B. M. \& Compagnon, I. Discrimination of patterns of $\mathrm{N}$-acetylation in 
chitooligosaccharides by gas phase IR spectroscopy integrated to mass spectrometry. Pure Appl. Chem. https://doi.org/10.1515/pac-2017-0110 (2017).

38. Masellis, C., Khanal, N., Kamrath, M. Z., Clemmer, D. E. \& Rizzo, T. R. Cryogenic vibrational spectroscopy provides unique fingerprints for glycan identification. J. Am. Soc. Mass Spectrom. https://doi.org/10.1007/s13361-0171728-6 (2017).

39. Mucha, E. et al. Glycan fingerprinting via cold-ion infrared spectroscopy. Angrew Chem. Int. Ed. https://doi.org/10.1002/anie.201702896 (2017).

40. Varki et al. Symbol nomenclature for graphical representation of glycans. Glycobiology 25, 1323-1324 (2015).

41. Ashline, J. et al. Carbohydrate structural isomers analyzed by sequential mass spectrometry. Anal. Chem. 79, 3830-3842 (2007).

42. Cord-Landwehr, S. et al. Quantitative mass-spectrometric sequencing of chitosan oligomers revealing cleavage sites of chitosan hydrolases. Anal. Chem. 89, 2893-2900 (2017).

43. Chambon, R. et al. Efficient chemoenzymatic synthesis of lipo-chitin oligosaccharides as plant growth promoters. Green Chem. 17, 3923-3930 (2015).

\section{Acknowledgements}

This work was supported by Université Lyon 1, Institut Universitaire de France and the Fédération de Recherche André Marie Ampère. This work was granted access to the HPC resources of the FLMSN, 'Fédération Lyonnaise de Modélisation et Sciences Numériques', partner of EQUIPEX EQUIP@MESO and to the 'Centre de calcul CC-IN2P3' at Villeurbanne, France. This collaborative work takes place within the Glycophysics Network (http://glyms.univ-lyon1.fr) with the support of the French Agence Nationale de la Recherche (Grant ANR-2015-MRSEI-0010)) and was supported in the UK by BBSRC: [sLoLa BB/K00199X/1] and BBSRC, EPSRC and InnovateUK: [IBCatalyst BB/ M02903411 and BB/M028836/1]. SF acknowledges support from ICMG FR 2607, Labex ARCANE (ANR-11-LABX-0003-62 01) and PolyNat Carnot Institute.

\section{Author contributions}

B.S. and G.R. did mass spectrometry and spectroscopic measurements. S.C., S.F., C.G. and S.F. did chemistry planning, chemical synthesis and biosynthesis. A.-R.A., L.B. and C.L. did ab initio simulations. I.C. supervised project. I.C. and B.S. defined analytical strategy and did article write-up.

\section{Additional information}

Supplementary Information accompanies this paper at doi:10.1038/s41467-017-01179-y.

Competing interests: The authors declare no competing financial interests.

Reprints and permission information is available online at http://npg.nature.com/ reprintsandpermissions/

Publisher's note: Springer Nature remains neutral with regard to jurisdictional claims in published maps and institutional affiliations.

(c) (i) Open Access This article is licensed under a Creative Commons Attribution 4.0 International License, which permits use, sharing, adaptation, distribution and reproduction in any medium or format, as long as you give appropriate credit to the original author(s) and the source, provide a link to the Creative Commons license, and indicate if changes were made. The images or other third party material in this article are included in the article's Creative Commons license, unless indicated otherwise in a credit line to the material. If material is not included in the article's Creative Commons license and your intended use is not permitted by statutory regulation or exceeds the permitted use, you will need to obtain permission directly from the copyright holder. To view a copy of this license, visit http://creativecommons.org/ licenses/by/4.0/.

(C) The Author(s) 2017 